\title{
High-speed laser-induced fluorescence and spark plug absorption sensor diagnostics for mixing and combustion studies in engines
}

\author{
Michael Cundy, ${ }^{1, *}$ Torsten Schucht, ${ }^{2}$ Olaf Thiele, ${ }^{2}$ and Volker Sick ${ }^{1}$ \\ 'Department of Mechanical Engineering, University of Michigan, W. E. Lay Automotive Laboratory, \\ 1231 Beal Avenue, Ann Arbor, Michigan 48109-2133, USA \\ ${ }^{2}$ LaVision GmbH, Anna-Vandenhoeck-Ring 19, D-37081 Göttingen, Germany \\ *Corresponding author: mcundy@umich.edu
}

Received 31 July 2008; revised 14 November 2008; accepted 19 November 2008;

posted 26 November 2008 (Doc. ID 99673); published 22 December 2008

\begin{abstract}
Simultaneous high-speed in-cylinder measurements of laser-induced fluorescence of biacetyl as a fuel tracer and mid-infrared broadband absorption of fuel and combustion products (water and carbon dioxide) using a spark plug probe are compared in an optical engine. The study addresses uncertainties and the applicability of absorption measurements at a location slightly offset to the spark plug when information about mixing at the spark plug is desired. Absorbance profiles reflect important engine operation events, such as valve opening and closing, mixing, combustion, and outgassing from crevices. (C) 2008 Optical Society of America

OCIS codes: $\quad 060.2370,120.1740,110.6915,300.1030,300.2530$.
\end{abstract}

\section{Introduction}

Spark ignition direct injection (SIDI) stratified charge engines offer significant fuel economy improvements when compared to conventional port fuel injected engines [1]. At full load, these engines are operated with a homogeneous charge like port fuel injected engines, but since liquid fuel is injected directly into the cylinder its evaporation leads to charge cooling. This allows higher loads and compression ratios to be used, which results in thermal efficiency gains. The brake-specific fuel consumption of these engines can approach that of diesel engines, without the associated noise or extra weight. Load is controlled by varying the amount of fuel injected, as opposed to varying the amount of fuel and throttling the intake air to create a homogeneous, flammable mixture. This requires that the mixture is suitably stratified to ensure ignition and complete combustion for low to mid load engine operation. A locally

0003-6935/09/040B94-11 $\$ 15.00 / 0$

(ㄷ) 2009 Optical Society of America rich region around the spark plug is desirable at the time of ignition to facilitate ignition and to promote early flame propagation because of its higher laminar flame speed. This is critical when operating an engine with overall high excess air or exhaust gas recirculation levels, which are approaches to minimize emissions. One particular engine concept that utilizes an inhomogeneous (stratified) mixture that has attracted considerable interest recently is the spray-guided (SG) SIDI stratified charge gasoline engine.

In general, proper mixture preparation in internal combustion engines is essential to simultaneously achieve maximum engine performance and minimized emissions. Cycle-to-cycle variations in combustion efficiency including partial burns and misfires are affected by ignition and early flame development near the spark plug, which are in turn largely affected by the local fuel/air/exhaust gas recirculation mixture at the spark plasma and in the regions surrounding the flame kernel. The processes involved vary spatially and temporally from one engine cycle to another. Studies of ignition and 
combustion under such conditions will require diagnostic tools that can resolve relevant scales. In particular, the rate at which spatially resolved measurements of fuel could be performed was limited until recently. This has motivated the development of high-speed laser-induced fluorescence (LIF) fuel imaging diagnostics [2] and absorptionbased spark plug optical probes [3, 4$]$. LIF measurements deliver images at adequately high rates but require the use of an optical engine. The images either exclude the third dimension or spatially average over it in the case of volume illumination. Aside from observing the fuel plume development and evaporation, statistical information can be extracted from quantitative LIF images using data analysis windows, and these cannot always be selected in ideal locations. Absorption-based spark plug sensors can be used in any engine with minimal or no modification and offer even higher measurement rates, but they lack spatial resolution. Especially under highly stratified fuel/air mixing situations, such as in a SG SIDI engine, the fuel concentration at the spark gap and the absorption path (typically a few millimeters away from the gap) may be quite different. The present paper evaluates the use of an optical spark plug probe in a SG SIDI engine. The probe measures infrared absorption of hydrocarbons, carbon dioxide, and water to derive fuel and product gas concentrations. Simultaneous measurements with the spark plug sensor and high-speed LIF were conducted in an optical SG SIDI engine at crank angle degree $(\mathrm{CAD})$ resolution $(3600 \mathrm{~Hz})$.

Comparison of the results of the two techniques allowed an evaluation of their precision when the engine was operated with a homogeneous fuel/air mixture. The higher signal-to-noise ratio of the absorption measurements allowed an assessment of the precision limitation of the LIF measurements, which are noise limited due to limited laser pulse energies at high repetition rates. LIF and absorbance data that were acquired under highly stratified conditions were normalized with data that were taken under homogeneous mixing conditions. This eliminated pressure, temperature, and number density effects on the signals, thereby allowing spatial and temporal analysis of the signals, which are measured millimeters from the spark gap in different locations.

\section{Background}

\section{A. Planar Laser-Induced Fluorescence}

Fuel imaging using LIF is accomplished by adding a fluorescence tracer to a nonfluorescing base fuel. A variety of tracer/fuel combinations and excitationdetection strategies are described in the literature [5]. For high-repetition-rate imaging only one tracer/fuel system (biacetyl/iso-octane) has been used [6]. Biacetyl has been selected to take advantage of the higher pulse energies that can be achieved at $355 \mathrm{~nm}$ compared to $266 \mathrm{~nm}$ using diode-pumped Nd:YAG lasers. For the $100 \mathrm{~ns}$ pulse duration and a sheet with a cross section of $20 \mathrm{~mm} \times 0.5 \mathrm{~mm}$ that was used in the present study the saturation pulse energy would be $\sim 4.3 \mathrm{~J}$. In this experiment, the maximum pulse energies approach $1 \mathrm{~mJ}$ so the LIF signal varies linearly with laser energy according to Eq. (1):

$$
S_{\mathrm{LIF}}=c I_{\text {Laser }}(\lambda) V\left(\frac{X_{\text {tracer }} p}{k T}\right) \sigma(\lambda, p, T, y) \varphi(\lambda, p, T, y),
$$

where $c$ is the overall efficiency of the collection optics; $I_{\text {Laser }}$ is the laser energy density; $V$ is the detection volume; the quantity $\left(\frac{X_{\text {tracer }} P}{k T}\right)$ represents the number density of the fluorescing species, determined by the mole fraction of the species of interest, $X_{\text {tracer }}$, the pressure, $P$, the Boltzmann constant $k$, and the temperature $T ; \sigma$ is the absorption cross section, $f$ is the fluorescence quantum yield; and $y$ is the mixture composition.

The photophysics of biacetyl for engine-relevant pressure and temperature conditions have been studied by Wermuth and Sick [7], Guibert et al. [8], and Smith and Sick [9]. Early engine biacetyl LIF experiments reported signal decay that was suggested to be caused by chemical decomposition at ambient conditions, but a dedicated species stability study by Zhang et al. [10] related that to sequential evaporation. Adequate chemical stability of biacetyl as a fluorescence tracer for fuel under spark-ignition conditions was found in a numerical study by Sick and Westbrook [11].

Some of the images presented in this work were recorded under late injected stratified charge conditions. In order to produce quantitative fuel concentration data the images have to be processed using background subtraction, light sheet division, and filtering to reduce noise. Recall that the available pulse energy was rather low and the fluorescence images will exhibit significant shot noise.

Background light, both $355 \mathrm{~nm}$ scatter and fluorescence from, e.g., surface deposits, is scattered by passing through windows and by reflecting off of features in the combustion chamber. Scattered $355 \mathrm{~nm}$ light is effectively absorbed by a GG420 filter. To eliminate remaining background signal from the images of interest, background images were taken at each $\mathrm{CAD}$ when the engine was motored (no fuel injection) for a statistically significant number of cycles $(\sim 100)$. Images taken at a given $\mathrm{CAD}$ were averaged and then subtracted from the light sheet and late injected images at the same CAD.

In order to generate quantitative data for stratified fuel images, reference LIF images can be used that were taken with a known fuel concentration, set by the fuel injection duration. This is accomplished by capturing LIF images in the compression stroke after a known amount of fuel is injected early in the intake stroke, producing quasi-homogeneous mixtures that correspond to a defined fuel concentration. These images then automatically include the effects of temperature, pressure, number density, 
and incongruities in the light sheet and detection path for each crank angle [9]. Light sheet images are taken in between late injected runs, so it is reasonable to assume that the laser sheet alignment and condition of the windows are sufficiently stable. It is assumed that temperature and pressure are homogeneous within the illuminated area. The division of the late injection images by the light sheet images resulted in quantitative fuel distribution maps.

The final processing step is spatial filtering by either a $3 \times 3$ averaging filter or an adjustable nonlinear anisotropic diffusion filter [12]. The latter filter is used to smooth out shot noise within flow structures while preserving large gradients.

The cumulative one standard deviation error of $17 \%$ for the LIF diagnostics is described in detail by Smith and Sick [9]. The use of higher laser power in the present study and spatial averaging allowed an improved uncertainty.

\section{B. Absorption Measurements}

In contrast to tracer-based measurements via LIF, infrared absorption allows the direct measurement of fuel as well as an aggregate of combustion product gases; in the case of this study carbon dioxide and water.

Molecular absorption is governed by the BeerLambert law. The absorbance is defined in Eq. (2):

$$
-\ln \left(\frac{I}{I_{0}}\right)=\sigma \cdot c \cdot L
$$

where $\sigma$ is the absorption coefficient, $L$ is the absorption path length, and $c$ is the density of the absorbing species. For the work presented here the absorption path length is constant. The absorption coefficient is assumed independent of temperature and pressure as a first approximation, but fully quantitative confirmation of this assumption is still pending, while the work presented here addresses this assumption to some extent. Additionally, the absorption conditions have to be assumed constant along the path length $L$. Then, the absorbing molecule density can be determined using Eq. (2).

Spectrally highly resolved measurements, e.g., using diode lasers, usually require information on wavelength-dependent absorption cross sections that are functions of temperature and pressure. The approach taken here is based on broadband absorption where the rotation structure of the absorption spectrum is not resolved. A broadband light source is used, and detection covers a large portion of the entire fundamental vibrational band. Integration over the entire rotational manifold is expected to minimize temperature sensitivity. However, vibrational population and line broadening effects due to pressure and temperature may still affect the absorption signal and eventually will need to be quantified. The broadband absorption strategy makes the measurement sensitive to cross talk with absorption by other molecules, although in the present case only to a small degree.

Absorption bands in the mid-IR are much stronger than the overtone bands that are often used in tunable diode laser absorption spectroscopy. A singlepass absorption setup with a path length of a few millimeters will then be sufficient to achieve a significant absorption that can be measured with a high signal-to-noise ratio. Broadband absorption measurements can be conducted with simple incandescent lamps such as tungsten halide mid-IR lamps.

Figure 1 shows the absorption spectrum for $\mathrm{CH}_{4}$ (representing hydrocarbon fuels), $\mathrm{CO}_{2}$, and $\mathrm{H}_{2} \mathrm{O}$ calculated from the HITRAN database [13]. Note that the absorption bands of hydrocarbon fuels and water overlap slightly. Potential cross talk of water absorption with fuel measurements may occur in cases where large amounts of water are present, although the highest water absorption coefficient is approximately two orders of magnitude lower in that spectral region. A HITRAN simulation for $600 \mathrm{~K}$ (the maximum temperature during our experiments) shows a cross talk of no more than $4 \%$.

A bandpass filter that coincides with the fundamental vibrational-rotational absorption band of hydrocarbons centered at $3.4 \mu \mathrm{m}(0.297 \mu \mathrm{m}$ width $)$ isolates light that is absorbed by the fuel. Biacetyl also has $\mathrm{C}-\mathrm{H}$ bonds and will absorb within this band, although the band may be slightly shifted because of the $\mathrm{C}=\mathrm{O}$ bonds. The net effect of adding biacetyl to iso-octane is not characterized. In general, even if it may be a good assumption to take the absorption coefficient as constant, which is checked as part of this study, the absolute magnitude of the absorption coefficient will depend on the molecular structure and composition of the fuel. A calibration measurement with a known fuel concentration is thus necessary. A bandpass filter centered at $2.7 \mu \mathrm{m}$ $(0.231 \mu \mathrm{m}$ width $)$ is used to measure the combined absorption by $\mathrm{CO}_{2}$ and $\mathrm{H}_{2} \mathrm{O}$. Note that at present, the temperature and pressure dependence of the absorption in this bandpass are not as well quantified as that of the fuel.

The high intensity of the IR lamp combined with sensitive detection allows measurements with high

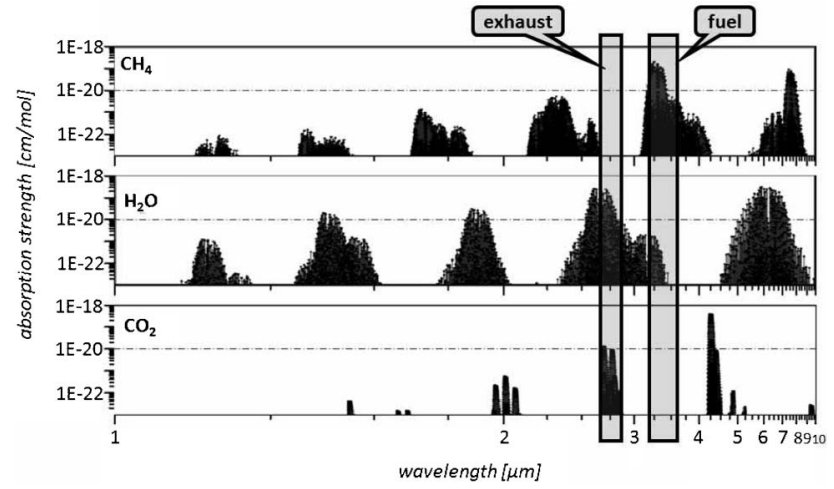

Fig. 1. Absorption spectrum of fuel, water, and carbon dioxide at $296 \mathrm{~K}$ The spectral simulations used the HITRAN database. 
signal-to-noise ratios with potentially significantly lower noise than single-shot LIF imaging. Absorption measurements can therefore be used here as a baseline to assess the precision of the highrepetition-rate LIF measurements.

\section{Experimental Setup}

As the goal of this work is an in-depth comparison of high-speed LIF and absorption-based in-cylinder measurements it is imperative that both measurements are conducted simultaneously. The presented measurements are a combination of high-speed CAD resolved planar LIF measurements recorded with a high-speed intensified camera and absorption measurements using an IR light spark plug sensor. Measurements were conducted in an optical singlecylinder engine with a four-valve head, 8-hole fuel injector, a transparent piston bowl, full quartz liner, and windows in the cylinder head. For the results presented below, the engine was run at $600 \mathrm{rpm}$ with the air intake temperature fixed at $45^{\circ} \mathrm{C}$. Moisture in the air intake is removed using filters. The air intake pressure was set at either 35 or $95 \mathrm{kPa} .10 \mathrm{mg}$ of fuel were injected with an end of injection of $250^{\circ}$ before top dead center (BTDC; CAD $=0$ at TDC) for the homogeneous experiments, and $40^{\circ} \mathrm{BTDC}$ for the stratified charge experiment. For fired engine operation, the spark timing was $30^{\circ} \mathrm{BTDC}$ (the others were motored). More details on the engine can be found in Table 1 and [14]. A schematic of the LIF diagnostics setup can be found in Fig. 2.

The signal-to-noise ratio of the LIF images is limited by the low pulse energy of high-repetition-rate lasers. For this experiment the combined beams of two frequency-tripled Nd:YAG lasers (Quantronix Hawk II) emitting at $355 \mathrm{~nm}$ were used to excite the fuel tracer biacetyl ( $10 \%$ by vol.), which was added to the iso-octane base fuel. The beams were made to overlap using a waveplate and a polarizing beam splitter cube and were fired simultaneously. High transmission at $532 \mathrm{~nm} /$ high reflectivity at $355 \mathrm{~nm}$ mirrors were used to both redirect the beams and remove remnants of frequency-doubled light. A cylindrical lens $(+1000 \mathrm{~mm})$ was used to assist in thinning the light sheet. A second cylindrical lens $(-100 \mathrm{~mm})$ was used to expand the beams in the ver-

Table 1. Optical Engine Parameters

\begin{tabular}{lc}
\hline \multicolumn{1}{c}{ Parameter } & Value \\
\hline Bore & $8.6 \mathrm{~cm}$ \\
Stroke & $8.6 \mathrm{~cm}$ \\
Connecting rod & $15.92 \mathrm{~cm}$ \\
Head volume & $62.24 \mathrm{~cm}^{3}$ \\
Displacement & $499.92 \mathrm{~cm}^{3}$ \\
Compression ratio & $9: 1$ \\
Intake valve open & $362^{\circ} \mathrm{BTDC}$ \\
Intake valve close & $126^{\circ} \mathrm{BTDC}$ \\
Exhaust valve open & $139^{\circ} \mathrm{ATDC}$ \\
Exhaust valve close & $373^{\circ} \mathrm{ATDC}$ \\
Valve lift & $10.3 \mathrm{~mm}$ \\
\hline
\end{tabular}

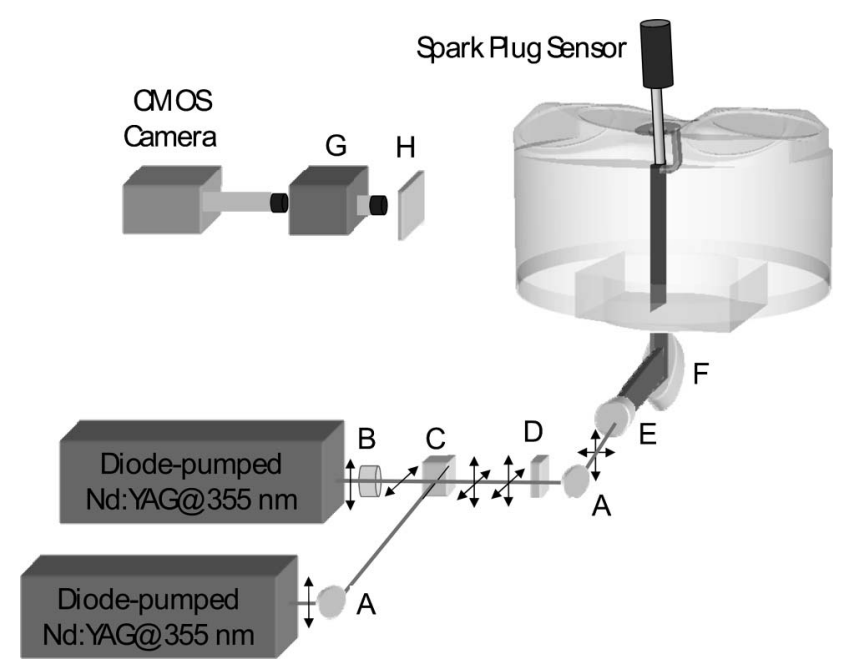

Fig. 2. Schematic of the experimental setup. The cylinder outline geometry is based on a computational grid (courtesy of Brian Peterson). The labeled optical elements are A\&F, dielectric mirror HT532/HR355; B, half-wave plate; C, beam splitter cube; D, cylindrical lens; E, cylindrical lens combination; G, high-speed image-intensified relay optics; H, GG420 color glass filter.

tical direction. A sheet-forming telescope (Rodenstock) was then used to focus the sheet. The sheet entered the engine between the crankcase and combustion chamber, where it reflected off of a $45^{\circ}$ mirror towards the cylinder head. It passed through the piston, which has a large axial window as well as a bowl in crown with side windows for viewing. This bowl is commonplace in SG SIDI engines along with an extended length spark plug to help confine the stratified fuel/air mixture around the spark plug and to initiate combustion near the center of the combustion chamber.

After traveling through the piston, the light sheet impinged on the cylinder head, extending from the fuel injector to slightly past the spark plug installation hole, bisecting the spark plug electrodes and absorption optical path. A schematic of the spark plug probe can be found in Fig. 3, and a schematic of the cylinder head showing the light sheet position and features within the combustion chamber can be seen in Fig. 4. Note that one of the eight fuel spray plumes is directed towards the spark plug. Also, note that the spark plug portion of the probe lies $\sim 2 \mathrm{~mm}$ in front of

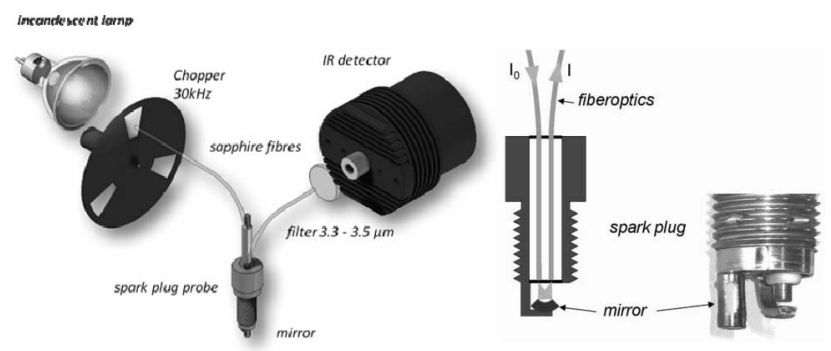

Fig. 3. Schematic of the spark plug probe. The left image is shown with one channel for illustrative purposes, and the right image shows the position of the absorption path relative to the spark plug electrodes. 

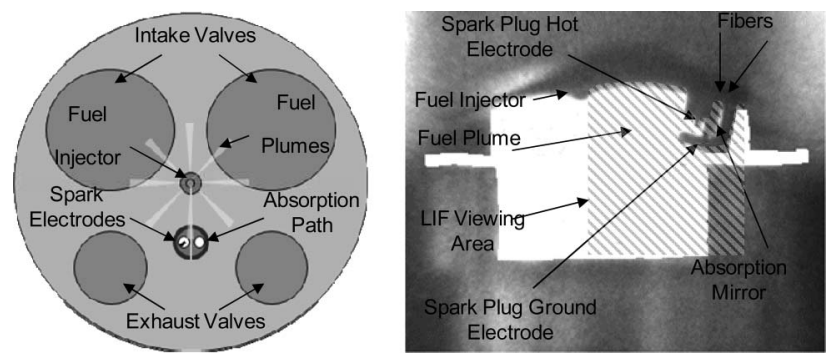

Fig. 4. Horizontal and vertical cross sections shown to illustrate the locations of the LIF and spark plug probe measurements relative to the spark plug electrodes. The LIF measurements are taken in the hatched area in the right image.

the light sheet and the absorption sensor portion lies behind the sheet by about the same distance. The spark plug probe package includes spacers of various thicknesses but cannot in its current form extend far enough into this engine for stable stratified charge operation with the standard injector. The sensor plug is a few millimeters shorter than the standard spark plug for this engine, and thus the fuel plume misses the spark plug. This alleviated any concern that liquid fuel accumulated in the absorption optical path during late injected runs.

Images were captured with a high-speed complimentary metal oxide semiconductor camera (Vision Research, Phantom v7.3). Signals were digitized at 14 bits and recorded until the $16 \mathrm{~GB}$ of on-board memory are filled. The weak LIF signals are amplified with a lens-coupled image intensifier (LaVision, IRO-HS). Synchronization of lasers, camera, and intensifier timing with the engine crank angle position was accomplished with a software-controlled highspeed timing unit [LaVision, High Speed Controller (HSC) and DaVis 7.2 software]. This also allowed adjustment of camera parameters and the setting of a gate on the camera and intensifier triggers to capture images only during the portions of the engine cycle of interest, enabling recording of hundreds of consecutive cycles.

The spark plug sensor system uses an incandescent (tungsten) lamp that is modulated at $30 \mathrm{kHz}$ using a chopper wheel. The modulation is required to record a background signal to account for background signal light that originates from the thermal radiation of the heated gases and straylight. The modulated light travels through a sapphire fiber to the spark plug, enters the cylinder through a sapphire window, is reflected by the mirror, and is transmitted back to the detection unit via a second fiber. The light is spectrally filtered and converted into an electrical signal using a two-stage TE-cooled IR photovoltaic detector (one for each channel). These analog signals were acquired with a 14 bit analogdigital converter (National Instruments) at a rate of $2.5 \mathrm{MHz}$. The chopper modulation reference signal and the crank angle encoder signals from the engine were recorded at the same rate. All these data were processed online in the sensor electronics to produce CAD resolved data and to split (demodulate) the traces into "light on" and "light off" signals. The data were transferred to a control computer via Ethernet, where three additional processing steps were performed to get the desired relative density traces. First, the background (light off) signal was subtracted from the light on signal, resulting in an absorption trace. Then the light intensity $I_{0}$ [see Eq. (1)] was determined dynamically for each individual cycle as the maximum recorded signal intensity in a given range where the fuel and product gas concentrations are at their lowest levels. The measurement of the reference light intensity through the entire absorption path allows accounting for any fouling of the mirrors or coupling lenses during engine operation. It also compensates for any potential intensity fluctuations of the incandescent lamp. In principle, $I_{0}$ should be measured in the absence of fuel (or exhaust for that channel). Splitting off a fraction of the incoming light and sending it through the same spectral filter bandpass as for the absorption detector would allow accounting for intensity fluctuations due to the lamp but not for window and mirror fouling. This would also require additional detectors. The impact on the accuracy of the absorption measurement when determining $I_{0}$ from the highest measured transmission could be assessed quantitatively. Approximately $11 \%$ of the gas volume will remain in the cylinder after the exhaust stroke. This amount is given by the compression ratio of the engine $(9: 1)$ and the exhaust backpressure. Thus, at any time during the cycle fuel absorption will be measured unless fuel injection is stopped for several cycles to purge the cylinder. Back-to-back measurements with and without fuel injection were conducted and $I_{0}$ was determined for both cases. The difference in measured absorbance, which is directly proportional to concentration, is less than $2 \%$. The absorbance measurement with $I_{0}$ determined dynamically is lower by this fraction.

Conventional engine parameters were recorded via a high-speed data acquisition system (National Instruments Compact DAQ controlled by Optimum Power software, PTrac) that allows recording of 28 channels at $\mathrm{CAD}$ resolution, digitized at 16 bits. The engine crank shaft position signals were first sent to the engine controller, where they were distributed to the spark plug sensor control and acquisition electronics and the data acquisition system mentioned above. The spark plug sensor electronics then relayed the signals to the HSC, which controlled the timing of the laser pulses, camera, and intensifier. All three systems were synchronized to record the same engine cycles.

\section{Results}

Results are presented on the precision, accuracy, and response to large spatial gradients for both measurement techniques. Simultaneously acquired absorption and LIF data are compared for this purpose for different mixing conditions. A discussion of absorption-based combustion product measurements 
that were carried out simultaneously with fuel measurements examines the use of broadband mid-IR absorption for aggregate water and carbon dioxide measurements.

\section{A. Homogeneous Charge Motored Measurements}

The signal-to-noise ratio of the absorption measurements [4] is expected to be higher than that of the LIF measurements [9], allowing the absorption data to be used as a benchmark for the precision of the LIF data. For this purpose, measurements in the motored engine were conducted with early fuel injection (end of injection at $250^{\circ} \mathrm{BTDC}$ ) to produce quasihomogeneous mixtures around the typical time of ignition. Figure $\underline{5}$ shows the results of this experiment, which also serve as light sheet images to normalize late injection images. An $11 \times 12$ pixel data analysis window corresponding to approximately $1.2 \times 1.3 \mathrm{~mm}$ was selected in each LIF image, the spatial average within the window was calculated, and the resulting values were averaged at their respective $C A D$ over 100 cycles. The location of the window is indicated in the inset picture on the plot. The ensemble standard deviation was also calculated for both the LIF and absorbance data and is indicated on the plot as variation bars. The images do not include any corrections (temperature, pressure, number density, etc.), and only a spatial $3 \times 3$ sliding filter has been applied. The spark plug probe measurements are presented in terms of absorbance $\left[-\ln \left(I / I_{0}\right)\right]$, corrected for background light. The LIF data axis was scaled arbitrarily to match the display of the absorbance data at $39^{\circ}$ BTDC.

In the absence of any temperature and pressure effects on the LIF and absorption signals the signals would vary inversely proportional to the in-cylinder

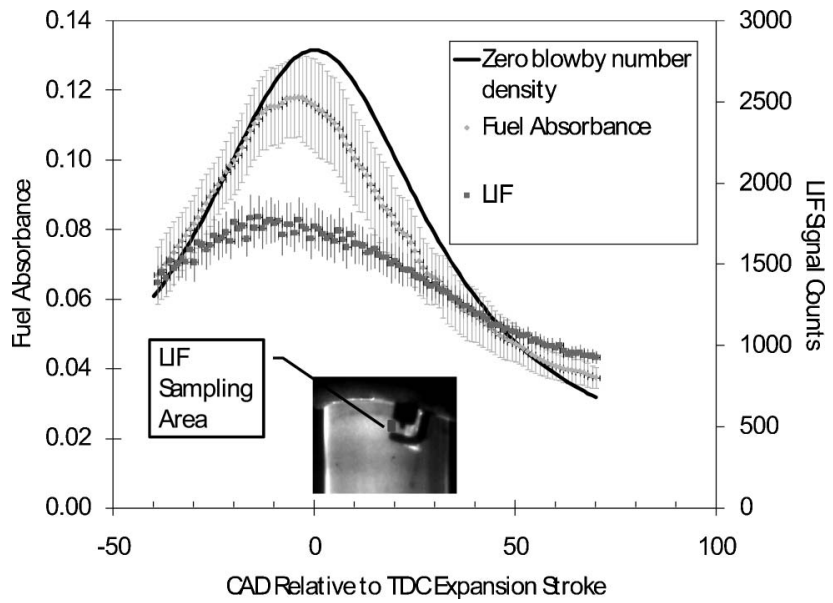

Fig. 5. Comparison of the spark plug probe absorbance measurements with the LIF signals in the homogeneous charge motored experiment. The LIF data are spatially averaged within the rectangle shown (132 pixels) and then averaged at each CAD over 100 cycles. The data contain one standard deviation variation bars. The data reflect the change number density as the chamber volume changes when the piston moves during the engine cycle. This change in the absence of engine blowby losses is shown as a solid curve. volume once the intake valves are closed $\left(126^{\circ}\right.$ BTDC). Thus, a comparison is presented in Fig. 5 to a scaled ideal number density value that is pegge $\bar{d}$ to the absorbance data at intake valve closing. It is noticeable that the absorbance data closely follow the expected number density, but starting at approximately $20^{\circ}$ BTDC the absorbance data fall below the expected values. The reason could either be a reduction of the absorption coefficient as pressure and temperature increase or mass loss by blowby across the nonlubricated piston rings. The observed magnitude of the signal reduction is commensurate with known significant blowby mass loss $(>10 \%)$ from the cylinder in nonlubricated optical engines [15]. The substantial mass loss is also the reason why peak signals for LIF and absorption are observed before the piston reaches TDC.

The LIF signal strength does not follow the increase in number density, pointing to a pronounced effect of temperature and pressure on the signal strength. The peak temperature during motored engine operation does not exceed $\sim 600 \mathrm{~K}$ [15] at a peak pressure of 15 bars. There is no evidence that biacetyl would be chemically decomposed under the experimental conditions of this study [11]. LIF signal strength for biacetyl after excitation at $355 \mathrm{~nm}$ was shown to drop with increasing pressure and temperature [9], and for the conditions shown in Fig. 5 the observed signal evolution is in good agreement with expectations. A full correction of the presented LIF signals for temperature and pressure effects would require accurate temperature data. For the given experiments, the engine exhibited unusually high blowby due to a slightly malfunctioning valve; this will cause a higher uncertainty in temperature analysis based on measured in-cylinder pressure traces. Therefore, only noncorrected LIF data for the homogeneous engine runs are presented.

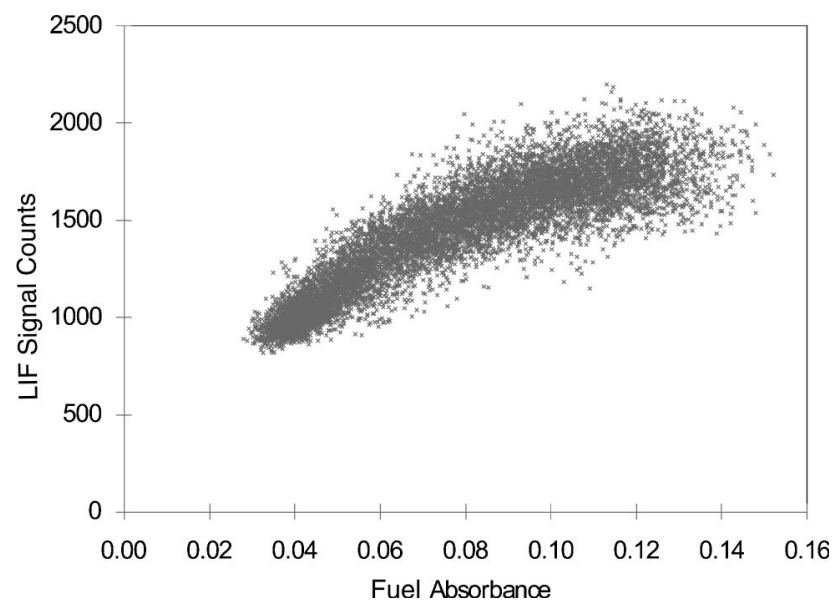

Fig. 6. Individual data from simultaneous LIF and absorption measurements for 100 cycles measured during the homogeneous charge motored experiment. The average and standard deviation of the same data are shown in Fig. $\underline{5}$ as a function of crank angle and illustrate the change in slope observed here as a pressure and temperature influence primarily on the LIF signal strength. 
The correlation of LIF and absorbance data are shown in Fig. 6 where individual data points from 100 cycles from $39^{\circ}$ BTDC to $70^{\circ}$ ATDC are plotted. The nonlinearity of this correlation reflects a difference in the temperature and pressure dependence of the signals. Recall that the LIF data have not been calibrated and have not been processed for temperature and pressure. A LIF signal decrease with increasing temperature and pressure is expected [9] and is observed in Fig. $\underline{6}$, as the highest LIF and absorption data were measured around TDC, where temperature and pressure are highest. This, along with the better correlation of the absorbance data with the in-cylinder volume before $20^{\circ} \mathrm{BTDC}$, supports the assumption of reduced temperature and pressure dependence of the IR fuel absorption coefficient.

An evaluation of the measurement precision is based on the standard deviation of the data from 100 consecutive engine cycles. To eliminate any influence of pressure and temperature on this analysis, the ensemble standard deviation values were normalized by the ensemble average at each CAD. For the LIF data a spatially averaged signal across 132 pixels was used from within a specified data analysis window as shown in Fig. 5. The results are shown in Fig. 7. The signal fluctuations for the two measurement techniques are initially higher, and then at TDC for the LIF signals and after TDC for absorbance data the variation decreases. The measured normalized standard deviation shows that homogeneity is increasing after TDC until fluctuations begin stabilizing at around 5\%. The noise level for absorbance was determined to be at or below $1 \%$ from transmissivity data $\left(I / I_{0}\right)$ from experiments without fuel injection. This marks the lower noise

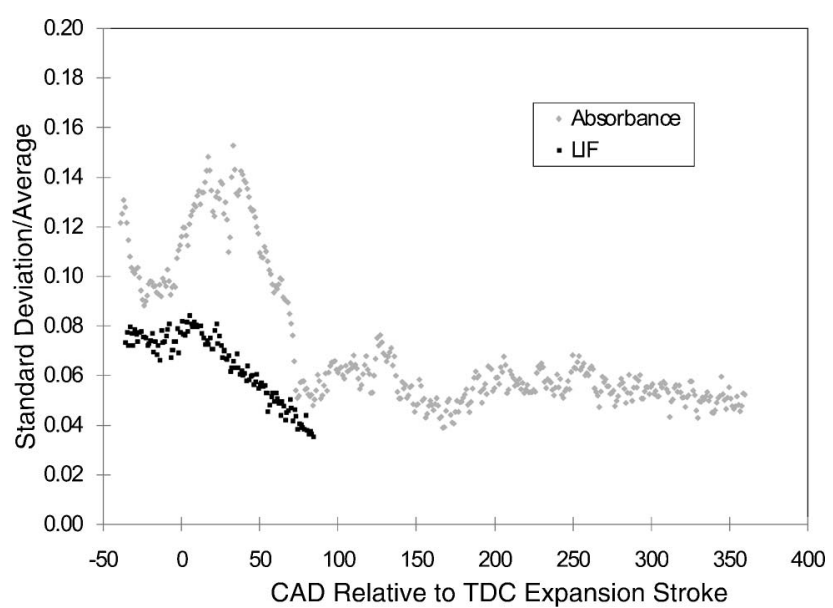

Fig. 7. Information on precision for LIF and absorption measurements from the homogeneous charge motored experiment is available from normalized standard deviation data. Ensemble standard deviation data from 100 consecutive engine cycles normalized by the average at their respective $\mathrm{CAD}$ are used to eliminate the influence of pressure and temperature dependence on the signals. Note that the noise limit of the absorption measurements was determined from runs without fuel injection to be $1 \%$ or less. LIF data are not available after $70 \mathrm{CAD}$. limit for the entire setup at the engine. The data confirm that local fluctuations in mixture homogeneity remain indeed at a level of 5\% for this engine operating condition.

\section{B. Stratified Charge Motored Measurements}

The ability to use the absorption measurement to deduce information about fuel concentration and its variation at the spark plug needs to be evaluated. This is important since the spark plug electrodes and absorption path are several millimeters apart from each other and spatial fuel gradients in SG SIDI engines can be very large. Correlations of simultaneously acquired LIF and absorbance data can be used for this evaluation. It is important to restate, though, that the LIF measurement volume is between the electrode gap and absorption optical path. Eliminating differences in LIF and absorption data that stem from temperature and pressure effects is important for this evaluation. Biacetyl LIF images from late injection engine runs were normalized with averaged LIF images from an early injection run. Similarly, absorbance data from late injection runs were normalized with averaged absorbance data from the same early injection run. The relative fuel concentrations that are reported from both measurements could be scaled to equivalence ratios or mole fractions after determining the amount of air present in the cylinder. Given the high blowby losses for the present experiment, we did not perform this scaling to avoid the introduction of a potential bias error that would depend on crank angle position. This would not prevent the evaluation and discussion of the fluctuations and the correlations between LIF and absorbance that was observed but it would also not add any relevant information to the present study, i.e., an assessment of local fluctuations and how different they are at the two measurement locations. Therefore, no absolute scaling to equivalence ratio or mole fraction was performed.

Processed LIF images (which have been corrected for temperature, pressure, number density, light sheet inhomogeneities, etc., as described above) from a single cycle during a late injected motored experiment are presented in Fig. 8. This particular image sequence was chosen to illustrate two points.

First, it is evident that the fuel misses the spark plug probe on its first pass and is recirculated from the piston bowl back to the cylinder head. From there, the amount of fuel present in the images appears to decrease, suggesting significant fuel motion in the third dimension or outside of the light sheet area. A fuel cloud reaches the data analysis window, shown in the image at $39^{\circ}$ BTDC just to the left of the spark gap, at approximately $19^{\circ}$ BTDC.

Second, a fuel droplet can be seen moving from the fuel injector to the spark plug in the earlier images. The in-cylinder flow in this engine is dominated by a strong tumble flow that is directed from the fuel injector to the spark gap [16]. Using the known temporal separation between images and the spatial scaling 


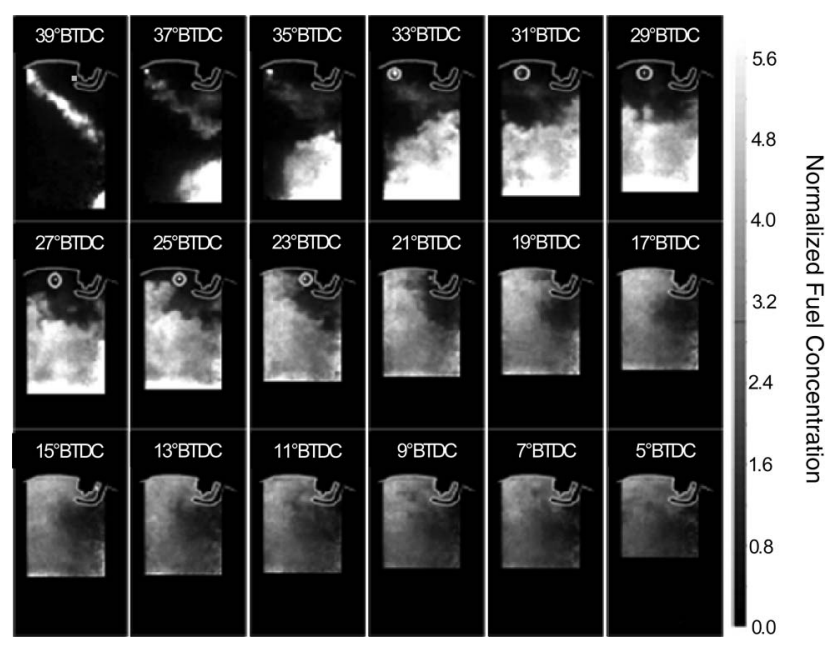

Fig. 8. Fully processed fuel concentration images from the late injected motored experiment. These images are used to visualize fuel plume development. Statistical information is also extracted from the data analysis window to the left of the spark electrodes (shown only in the $39^{\circ} \mathrm{BTDC}$ image for illustrative purposes) and is compared to the absorbance measurements. In images at $33-23^{\circ} \mathrm{BTDC}$, circles are placed in the images to the left of the spark plug to identify a fuel droplet which moves from left to right with the flow field (see text for more details.)

it was determined that this droplet is moving at approximately $2 \mathrm{~m} / \mathrm{s}$. The separation of the LIF data analysis window and the absorption path is $\sim 6 \mathrm{~mm}$. This suggests that the fuel will show up in the LIF images approximately 9-10 CADs before it registers in the absorbance measurements. Figure 9 shows a fuel concentration profile that was extracte $\bar{d}$ from images in the same cycle along with normalized absorbance data for the cycle. LIF data prior to $25^{\circ}$ BTDC have been excluded from the plot since an initial offset error in the LIF acquisition rendered images taken prior to this $\mathrm{CAD}$ nonquantitative. A

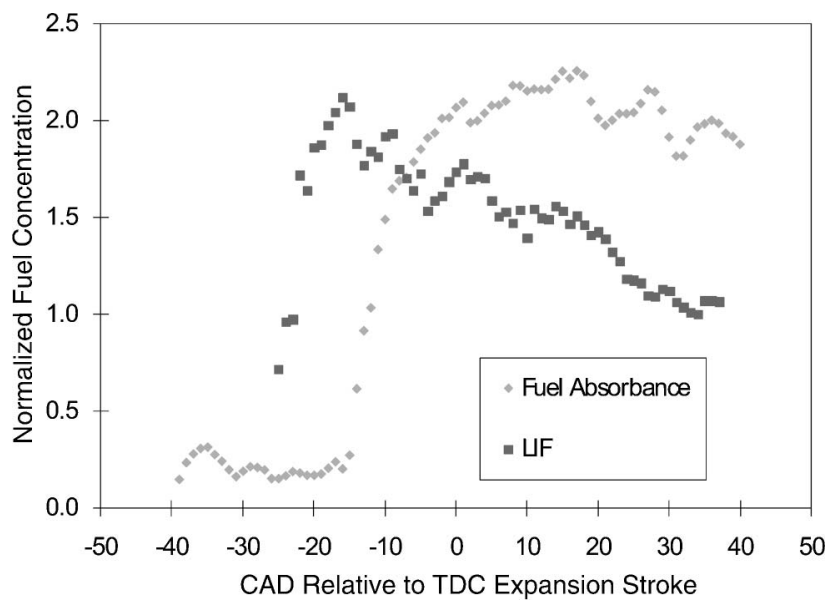

Fig. 9. LIF and absorbance probe fuel concentrations from the cycle shown in Fig. 8 from the late injected motored experiment. The LIF measurement shows the presence of fuel approximately $8 \mathrm{CADs}$ before fuel registers in the absorbance data. This delay is expected from the spatial separation of LIF measurement window and spark plug sensor based on the flow direction that was evident from the droplet motion shown in Fig. 8 . temporal shift in the rise of the fuel concentration at the absorbance measurement location is noticeable in the profiles, although to a lesser degree than estimated. Variations on the delay are expected from cycle to cycle since the in-cylinder flow is highly variable. This is especially true near obstructions such as the spark electrodes and the absorption mirror extrusion. The systematic delay in arrival of the fuel cloud at the spark plug sensor is longer in the average data shown in Fig. 10. This delay corresponds more closely with the estimated velocity of the droplet. The significant discrepancies between the normalized LIF and absorbance probe signals is likely due to the different probe measurement locations. One of the fuel plumes was directed towards the axis of the spark plug installation hole. The light sheet was aligned between the spark electrodes, and the absorption path and therefore this fuel plume almost fully lies within the path of the fuel spray. Conversely, the absorption path is about $2 \mathrm{~mm}$ away from the light sheet, so the LIF data were expected to show different dynamics.

An additional observation can be made in the absorbance data set shown in Figs. 9 and 10. Before fuel from the current injection arrives at the measurement location, greater than zero concentrations were measured. There is an expected residual amount of fuel in the cylinder from the previous cycle since the engine is just motored.

Figure 11 shows the results of this late injected experiment in scatter plot form. Data from $25^{\circ}$ BTDC to $70^{\circ}$ ATDC are included in this plot. There are striking differences noticed in comparison to the same assessment of the data from the homogeneous mixture experiment. The large fuel gradients, shown in the images in Fig. $\underline{8}$, suggest that there are similar gradients between the location of the LIF measurement volume and the absorption path. The result is that a

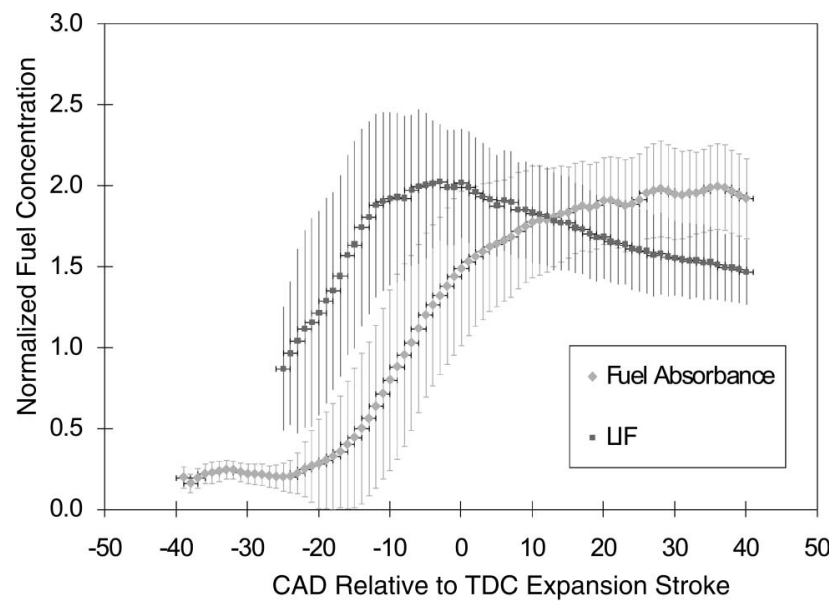

Fig. 10. 100 cycle average of quantitative LIF and absorbance data from the late injected motored experiment normalized by data from the homogeneous charge motored experiment to calibrate the signals (see text for details on calibration). The systematic delay in the arrival of fuel at the spark plug probe is consistent with estimated droplet velocities that were extracted from LIF image sequences. 


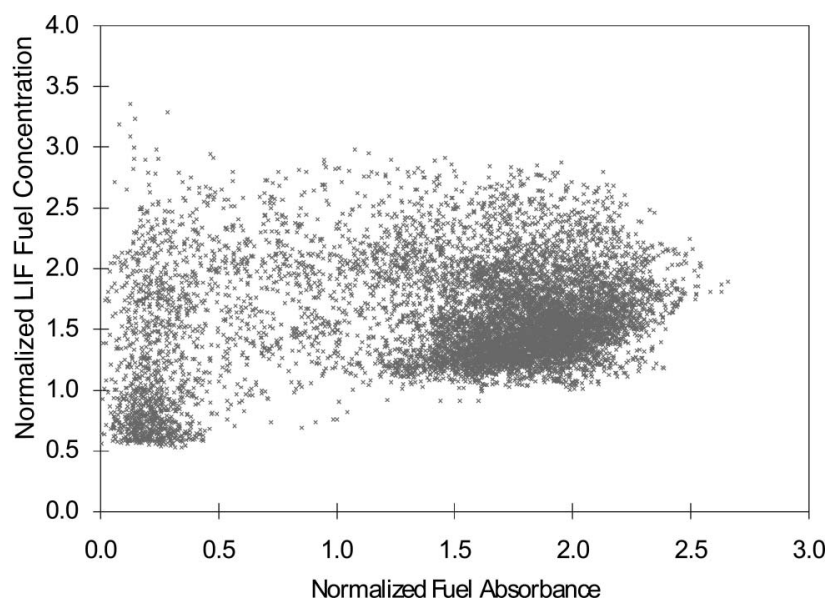

Fig. 11. Individual, simultaneously acquired LIF and absorption data points that were used to generate the average values and standard deviation bars in Fig. 10 are presented. The correlation is much weaker for stratified engine operation than for homogeneous mixing conditions. See Fig. 5 for comparison. The large spatial and temporal fuel gradients in fuel concentrations are manifested in this plot.

low correlation is expected, and this is clearly confirmed. It is noteworthy to point out that the LIF data are not expected to drop down to zero values; the images in Fig. 8 representatively show that at this time we already observe fuel at the LIF detection volume, while it is plausible that the absorption path does not yet have significant levels of fuel in it. The interpretation of either the LIF or absorption signal under highly stratified conditions must take into consideration that neither measurement location may coincide with the location of the spark plasma channel. However, overall timing and fluctuation information can be obtained from both types of signals.

\section{Homogeneous Charge Fired Experiments}

While the LIF measurement delivers information on the fuel concentration only, the spark plug sensor measures fuel and combustion product gas concentration (aggregate of $\mathrm{H}_{2} \mathrm{O}$ and $\mathrm{CO}_{2}$ ) simultaneously. This capability was demonstrated for an early injected engine run with the intake air throttled down to $\sim 35 \mathrm{kPa}$ at $45^{\circ} \mathrm{C}$ and a lean fuel/air mixture $(\phi \sim 0.8)$. The intake air was throttled to achieve near stoichiometric conditions since the quartz cylinder is unable to withstand running at full load (atmospheric intake conditions and $\phi \sim 1$ ). The spark was triggered at $30^{\circ}$ BTDC. This spark timing is somewhat early for optimized combustion conditions of the engine when running at $600 \mathrm{RPM}$ and thus higher than usual levels of unburned fuel were expected. The plasma luminosity is clearly visible from $29-$ $22^{\circ}$ BTDC in the LIF images shown in Fig. 12. The flame kernel likely begins in between the light sheet and the camera (see Fig. 4 for an illustration of the light sheet location) and eventually burns fuel within the light sheet beginning at $22^{\circ} \mathrm{BTDC}$.

Figure 13 shows the evolution of the LIF and absorbance signals that were recorded for the single cy-

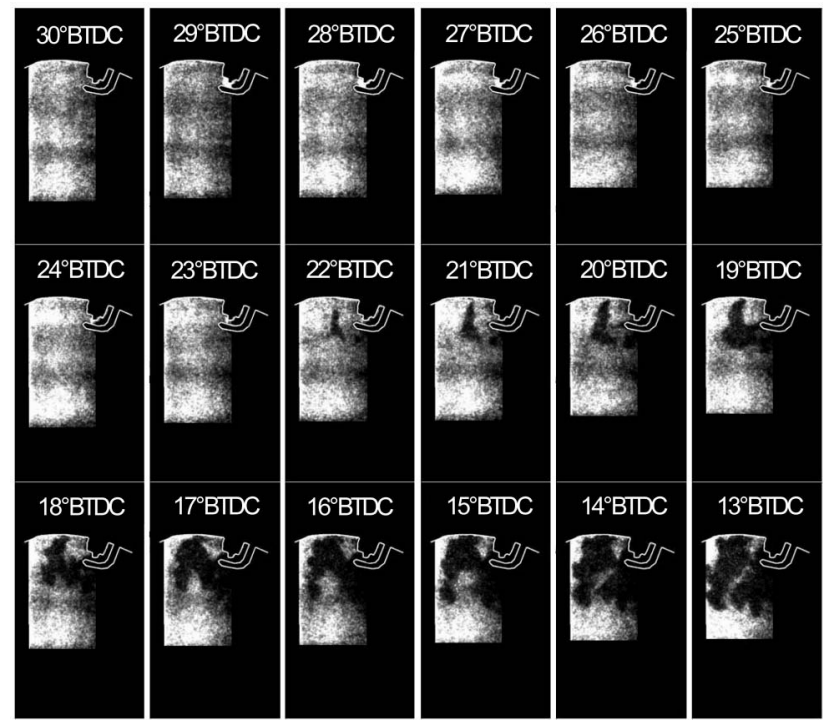

Fig. 12. Single-cycle LIF image sequence from a homogeneous charge part load combustion experiment. Note that the luminosity of the spark plasma is visible from $29-22^{\circ} \mathrm{BTDC}$. The biacetyl is consumed in the flame showing the corrugated growth of the flame front starting at $22^{\circ} \mathrm{BTDC}$.

cle shown in Fig. 12. The fuel absorbance rises from $60-15^{\circ}$ BTDC due to compression, as was observed in the motored measurements shown in Fig. 5. The LIF data for this randomly selected cycle show considerable scatter at the measurement location to the left of the spark plug. Fuel is not consumed at the measurement locations until $\sim 17^{\circ}$ BTDC, where each signal begins to drop and combustion product gas absorbance rises. The delay between the onset of the spark and the appearance of the flame in the measurement locations is likely due to the time required for energy transfer to the fuel and for the time for the flame kernel to develop. The LIF signal drops off more quickly than the fuel absorbance

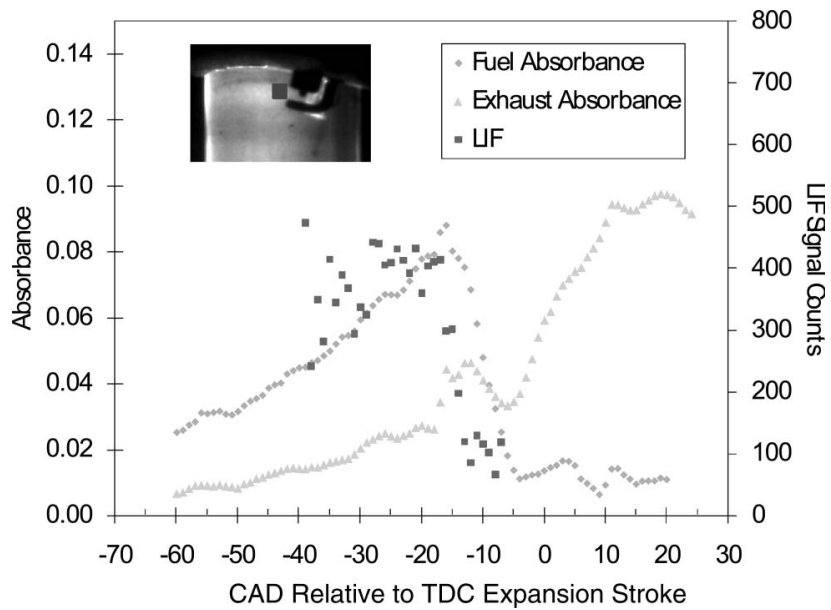

Fig. 13. Single-cycle LIF, fuel, and combustion product absorbance measurements from the homogeneous charge part load combustion experiment. The LIF data were extracted from the images shown in Fig. 12. Note that the location of the LIF data analysis window has been moved to avoid capturing spark luminosity. 
signal because the LIF measurement location is closer to the spark gap where the flame must have started. Note that the combustion product absorbance data clearly indicate the presence of residual gas in the cylinder from the previous cycle. Although no correction has been applied to determine the magnitude of these data, it is above the noise floor of the measurements and can be concluded to be residual gas. The absorbance signal of the (residual) product gasses also increases during compression along with the fuel signal because of increasing number density. Once combustion starts the signal actually first drops due to expansion of the (hot) combustion gas, and then signals increase again due to continued compression and combustion.

Figure 14 shows full cycle profiles of the results of fuel and product gas absorbance measurements averaged over 200 cycles. Both traces start out at residual gas levels and then drop considerably as they are diluted with fresh, dry intake air. Fuel was injected at $250^{\circ}$ BTDC and began to arrive at the spark plug sensor around $200^{\circ}$ BTDC. As mentioned before, the spark was triggered at $30^{\circ}$ BTDC. Similarly to what was observed in the single cycle shown in Fig. 13, on average the flame does not reach the measurement area until several CADs after the spark is triggered. The product gas absorbance trace shows a small hump near $15^{\circ}$ BTDC. This reflects the expansion of the gases due to combustion as discussed for the individual cycle above. The fuel trace also shows evidence of outgassing as fuel forced into crevices during compression is released during the expansion stroke without immediately oxidizing completely. This fuel eventually reached the probe around $30^{\circ} \mathrm{ATDC}$. After the exhaust valves open at $139^{\circ}$ ATDC fuel and exhaust signals began to rise and peak approximately $28 \mathrm{CADs}$ later. The cylinder pressure when the exhaust valves opened was $\sim 65 \mathrm{kPa}$, while the pressure in the exhaust manifold during the exhaust stroke was $\sim 96 \mathrm{kPa}$, so this peak is attributed to residual fuel and exhaust gases from the previous cycle flow-

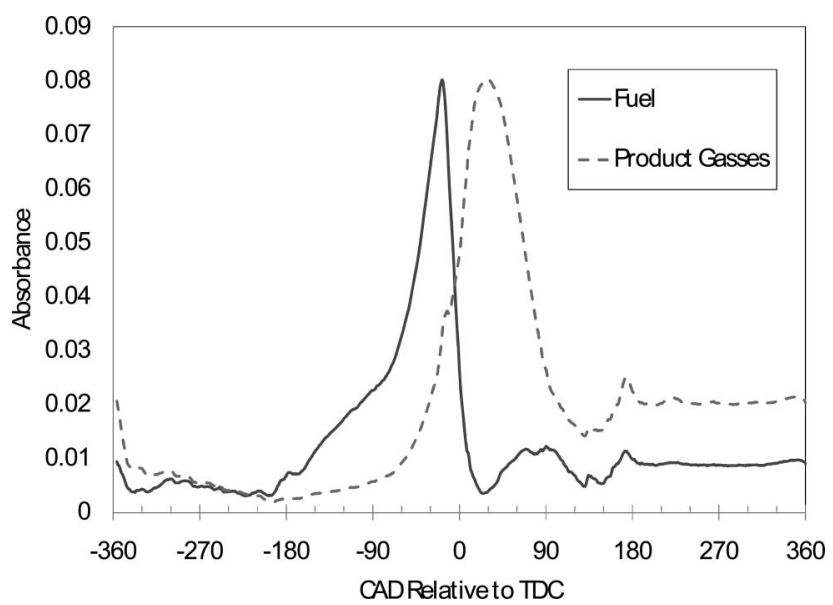

Fig. 14. Fuel and product gas absorbance signals averaged over 200 consecutive engine cycles. The traces show important engine operation characteristics such as ignition, outgassing, gas dynamics, and residual gas levels. ing back into the cylinder. The background (light off) signal from the spark plug probe was flat in this region, indicating actual gas dynamics and not some spurious input.

\section{Conclusions}

The use of optical diagnostics has assisted the design and development of modern internal combustion engines significantly. Now, more than ever, detailed temporally and spatially resolved information of mixing and combustion processes is needed to allow further improvements in the efficiency and the reduction of pollutant formation. This work describes simultaneous high-speed in-cylinder measurements of fuel concentration distributions using laserinduced fluorescence and fuel and exhaust gas concentrations using a mid-infrared absorption probe that was integrated into a spark plug. LIF measurements were based on biacetyl fluorescence that was induced by two simultaneously fired diode-pumped frequency-tripled Nd:YAG lasers. The broadband but spectrally filtered mid-IR measurements used the fundamental $\mathrm{C}-\mathrm{H}$ stretch absorption band of hydrocarbons to detect fuel near $3.4 \mu \mathrm{m}$, and combined absorption by water and carbon dioxide near $2.7 \mu \mathrm{m}$ was used as a measure for combustion product gases.

The combination of both techniques allowed a mutual assessment of the precision and accuracy of the measurements and the applicability of a pathintegrating absorption measurement at a location slightly offset to the spark plug when information about mixing at the spark plug is desired under highly stratified conditions.

The precision of the LIF measurements has been improved over previous work due to spatial averaging over 132 pixels and the use of higher laser pulse energies. Precision values as low as $4 \%$ are shown for a measurement with highest homogeneity during the exhaust stroke. However, the absorption measurements with a precision of better than $1 \%$ also show 4-6\% fluctuations in the fuel signal, indicating that this is a true fluctuation for the given operating conditions.

The analysis of the correlation of fuel absorbance and LIF signal intensity revealed that the assumption of a temperature and pressure independence of the mid-IR fuel absorption coefficient was reasonable around TDC for the iso-octane/biacetyl mixture that was used in the present study. A systematic time delay between LIF data taken from $a \sim 1 \mathrm{~mm}^{2}$ area near the spark plug and the fuel absorbance measurement for highly stratified engine operation was linked quantitatively to the fuel motion across the cylinder. No correlation between the LIF and absorbance values was present in this experiment due to this time delay and large spatial gradients in the fuel concentration, but the absorption probe can be used by itself to identify the arrival of a fuel cloud in the measurement path. Magnitudes of measured normalized fuel concentrations are matched for LIF and absorption, thus the temporally resolved 
absorption data can be used for parametric studies of near-spark plug events.

The simultaneous recording of fuel LIF and fuel and combustion products (aggregate of water and carbon dioxide) absorption during homogeneous combustion provided information about combustion onset and propagation as well as in-cylinder residual gases. Absorbance profiles reflect important engine operation events, such as valve opening and closing, mixing, combustion, and outgassing from crevices.

While the spark plug sensor measurements presented here indicate that the absorption coefficient for fuel (iso-octane/biacetyl) is less sensitive to pressure and temperature than biacetyl LIF, an absolute calibration strategy for absorption measurements requires quantitatively measured absorption coefficients. An additional detector should be added as a reference channel in a spectral region without absorption by fuel and exhaust gases (around $3 \mu \mathrm{m}$ ). This channel allows for a true $I_{0}$ measurement without assuming that at some time during the engine cycle absorption is negligibly small. This will especially be required to measure absolute exhaust gas recirculation rates. Exhaust gas measurements could also be based on $\mathrm{CO}_{2}$ absorption $(4.3 \mu \mathrm{m})$ to facilitate absolute calibration independent of the ratio of $\mathrm{CO}_{2}$ and $\mathrm{H}_{2} \mathrm{O}$.

The present study shows that a mid-IR absorption based spark plug probe simultaneously provides crank angle resolved data of relative fuel and exhaust gas densities near the spark gap. The data correlate well with the characteristics of data from highspeed LIF imaging. In contrast to LIF, absorption does not provide spatial concentration distributions, but it allows measurements in nonmodified engines.

This work was supported in part by General Motors R\&D within the GM-University of Michigan Collaborative Research Laboratory on Engine Systems Research at The University of Michigan.

\section{References}

1. F. Zhao, M.-C. Lai, and D. L. Harrington, "Automotive sparkignited direct-injection gasoline engines," Prog. Energy Combust. Sci. 25, 437-562 (1999).

2. J. D. Smith and V. Sick, "Crank-angle resolved imaging of fuel distribution, ignition and combustion in a direct-injection spark-ignition engine," SAE Transactions Journal of Engines 114, 1575-1585 (2005).

3. M. Koenig and M. J. Hall, "Cycle-resolved measurements of precombustion fuel concentration near the spark plug in a gasoline SI engine," SAE paper 981053 (Society for Automotive Engineering, 1998).

4. T. Berg, V. Beushausen, O. Thiele, and H. Voges, "Fibre optics spark plug sensor for the optimisation of engine combustion processes," Motortech. Z. 67, 2-6 (2006).

5. C. Schulz and V. Sick, "Tracer-LIF diagnostics: quantitative measurement of fuel concentration, temperature and fuel/ air ratio in practical combustion systems," Prog. Energy Combust. Sci. 31, 75-121 (2005).

6. J. D. Smith and V. Sick, "Crank-angle resolved imaging of biacetyl laser-induced fluorescence in an optical internal combustion engine," Appl. Phys. B 81, 579-584 (2005).

7. N. Wermuth and V. Sick, "Absorption and fluorescence data of acetone, 3-pentanone, biacetyl, and toluene at engine-specific combinations of temperature and pressure," SAE Trans. J. Fuels Lubricants 114, 804-814 (2005).

8. P. Guibert, V. Modica, and C. Morin, "Influence of pressure, temperature and gas phase composition on biacetyl laser-induced fluorescence," Exp. Fluids 40, 245-256 (2005).

9. J. D. Smith and V. Sick, "Quantitative, dynamic fuel distribution measurements in combustion-related devices using laserinduced fluorescence imaging of biacetyl in iso-octane," Proc. Combust. Inst. 31, 747-755 (2007).

10. R. Zhang, S. V. Bohac, and V. Sick, "Stability of iso-octane mixtures with 3-pentanone or biacetyl as fluorescence tracers in combustion experiments," Exp. Fluids 40, 161-163 (2006).

11. V. Sick and C. K. Westbrook, "Diagnostic implications of the reactivity of fluorescence tracers," Proc. Combust. Inst. doi: 10.1016/j.proci.2008.05.012.

12. P. Perona and J. Malik, "Scale-space and edge detection using anisotropic diffusion," IEEE Trans. Pattern Anal. Mach. Intell. 12, 629-639 (1990).

13. L. S. Rothman, D. Jacquemart, A. Barbe, D. C. Benner M. Birk, L. R. Brown, M. R. Carleer, C. C. Jr., K. Chance, L. H. Coudert, V. Dana, V. M. Devi, J.-M. Flaud, R. R. Gamache, A. Goldman, J.-M. Hartmann, K. W. Jucks, A. G. Maki, J.-Y. Mandin, S. T. Massie, J. Orphal, A. Perrin, C. P. Rinsland, M. A. H. Smith, J. Tennyson, R. N. Tolchenov, R. H. Toth, J. V. Auwera, P. Varanasi, and G. Wagner, "The HITRAN 2004 molecular spectroscopic database,” J. Quant. Spectrosc. Radiat. Transf. 96, 139-204 (2005).

14. U. Fissenewert, V. Sick, and H. Pucher, "Characterization of combustion and NO formation in a spray-guided gasoline direct-injection engine using chemiluminescence imaging, NOPLIF, and fast NO exhaust gas analysis," SAE Trans. J. Fuels Lubricants 114, 786-803 (2005).

15. W. Koban, J. D. Koch, V. Sick, N. Wermuth, R. K. Hanson, and C. Schulz, "Predicting LIF signal strength for toluene and 3-pentanone under engine-related temperature and pressure conditions," Proc. Combust. Inst. 30, 1545-1553 (2005).

16. C. M. Fajardo and V. Sick, "Flow field assessment in a fired spray-guided spark-ignition direct-injection engine based on UV particle image velocimetry with sub crank angle resolution," Proc. Combust. Inst. 31, 3023-3031 (2007). 